\title{
Kidney transplantation recovers the reduction level of serum sulfatide in ESRD patients via processes correlated to oxidative stress and platelet count
}

Lixuan Wang a, b, 1 , Yuji Kamijo ${ }^{\text {a, c, }}{ }^{*}, 1$, Akihiro Matsumoto ${ }^{\text {d, Takero Nakajima }}{ }^{\text {a }}$, Makoto

Higuchi $^{\mathrm{c}}$, Reiji Kannagi ${ }^{\mathrm{e}}$, Mamoru Kyogashima ${ }^{\mathrm{f}, \mathrm{g}}$, Toshifumi Aoyama ${ }^{\mathrm{a}}$, Atsushi Hara ${ }^{\mathrm{a}}$

${ }^{a}$ Department of Metabolic Regulation, Institute on Aging and Adaptation, Shinshu University Graduate School of Medicine, 3-1-1 Asahi, Matsumoto, Nagano 390-8621, Japan

${ }^{\mathrm{b}}$ Department of Histology and Embryology, Hebei Medical University, 361 Zhong Shan East Road, Shijiazhuang, Hebei 050017, China

${ }^{\mathrm{c}}$ Department of Nephrology Internal Medicine, Shinshu University School of Medicine, 3-1-1 Asahi, Matsumoto, Nagano 390-8621, Japan

${ }^{\mathrm{d}}$ Department of Hepatology Internal Medicine, Shinshu University School of Medicine, 3-1-1 Asahi, Matsumoto, Nagano 390-8621, Japan

${ }^{\mathrm{e}}$ Research Complex for the Medicine Frontiers, Aichi Medical University, 21 Karimata, Yazako, Nagakute, Aichi 480-1195, Japan

${ }_{\mathrm{f}}^{\mathrm{f}}$ Division of Molecular Pathology, Aichi Cancer Center Research Institute, 1-1 Kanokoden, Chikusa-ku, Nagoya 464-8681, Japan

${ }^{g}$ Department of Oncology, Graduate School of Pharmaceutical Sciences, Nagoya City University, 3-1 Tanabe-dori, Mizuho-ku, Nagoya 467-8603, Japan

${ }^{*}$ Corresponding author: Yuji Kamijo, M.D., Ph.D.

Department of Metabolic Regulation, Institute on Aging and Adaptation, Shinshu University Graduate School of Medicine, 3-1-1 Asahi, Matsumoto 390-8621, Japan

Phone: +81-263-37-2850; Fax: +81-263-37-3094; E-mail: yujibeat@shinshu-u.ac.jp

${ }^{1}$ The first two authors contributed equally to this work. 


\section{Abstract}

Sulfatide is a major component of glycosphingolipids in lipoproteins. Recently, we reported that a low serum level of sulfatide in hemodialysis patients might be related to the high incidence of cardiovascular diseases. However, the serum kinetics of sulfatide in kidney disease patients and the function of endogenous serum sulfatide are still unclear. To obtain novel knowledge concerning these issues, we investigated the serum kinetics of sulfatide in 5 adult kidney transplant recipients. We also analyzed the correlated factors influencing the serum sulfatide level, using multiple regression analysis. Kidney transplantation caused a dramatic increase of serum sulfatide without an alteration of its composition in all recipients in a time-dependent manner; however, the recovery speed was slower than that of the improvement of kidney function and the serum sulfatide reached a nearly normal level after 1 year. Multiple regression analysis showed that the significant correlated factor influencing the serum sulfatide level was log duration (time parameter) throughout the observation period, and the correlated factors detected in the stable phase were the decrease of serum concentration of malondialdehyde (an oxidative stress marker) as well as the elevation of platelet count. The current study results demonstrated the gradual but reliable recovery of the serum sulfatide level in kidney transplant recipients for the first time, suggesting a close correlation between serum sulfatide and kidney function. The recovery of serum sulfatide might derive from the attenuation of systemic oxidative stress. The normal level of serum sulfatide in kidney transplant recipients might affect platelet function, and contribute to the reduction of cardiovascular disease incidence. 
Key words: kidney transplantation, oxidative stress, platelet, serum sulfatide

\begin{abstract}
Abbreviations: AKI, acute kidney injury; CST, cerebroside sulfotransferase; CVD, cardiovascular disease; ESRD, end-stage renal disease; GP, glycoproteins; HD, hemodialysis therapy; LS, lysosulfatide; d18:2, sphingadienine; d18:1, (4E)-sphingenine; d18:0, sphinganine; t18:0, phytosphingosine; d20:1, (4E)-icosasphingenine; d20:0, icosasphinganine; t20:0, 4D-hydroxyicosasphinganine; MALDI-TOF MS, matrix-assisted laser desorption ionization-time of flight mass spectrometry; MDA, malondialdehyde; $\mathrm{PD}$, peritoneal dialysis therapy; $\mathrm{SD}$, standard deviation; vWF, von Willebrand factor
\end{abstract}




\section{Introduction}

Sulfatide is an ester of sulfuric acid with galactosylceramide at $\mathrm{C} 3$ of the galactosyl residue, which is widely distributed in various organs, including the kidney, nervous system, liver, and digestive tract [1-3]. Sulfatide is also present in mammalian serum as a major component of glycosphingolipids in lipoproteins $[4,5]$. The authors of many earlier studies had reported that serum sulfatide exerted considerable effects on atherosclerosis, coagulation, and platelet function [6]. A series of studies using an animal model for human familial hypercholesterolemia showed the marked increase of sulfatide in the sera and atherosclerotic plaques, suggesting their participation in atherosclerosis $[4,7]$. The results of various experimental studies investigating the effects of the administration of exogenous sulfatide showed that serum sulfatide exerts anti-coagulant and anti-platelet effects $[6,8]$. However, there has been little information concerning the function of endogenous serum sulfatide in humans or exploring the relationship between the serum level of sulfatide and human diseases.

Recently, we reported that the serum level of sulfatide in end-stage renal disease (ESRD) patients undergoing hemodialysis therapy (HD) was significantly lower than that in age-matched normal subjects [9]. This earlier study demonstrated that the reduction of the serum sulfatide level in ESRD patients was detected prior to the induction of HD treatment, and that the reduction level linearly decreased according to HD duration. Therefore the kidney dysfunction per se appeared to decrease the serum sulfatide level, which was exaggerated due to the long HD duration. This retrospective observational study also revealed that a low level of serum sulfatide was related to the incidence of cardiovascular diseases (CVD) in these patients [9]. This clinical research suggested the close relation between the serum level of sulfatide and kidney function, and the potential of sulfatide to be one of the inhibitory factors of CVD. To investigate the serum kinetics of sulfatide under kidney dysfunction, we 
conducted another study using a murine model of acute kidney injury (AKI), protein overload nephropathy [10]. This animal study demonstrated that acute kidney dysfunction dramatically reduced the serum level of sulfatide, like that of ESRD patients. This experiment also revealed that the reduction of serum sulfatide might be derived from the systemic enhancement of oxidative stress via kidney dysfunction. However, the serum kinetics of sulfatide in kidney disease patients and the function of endogenous serum sulfatide are still unclear.

To obtain novel knowledge about the alteration of serum sulfatide via kidney dysfunction in humans, we investigated the serum kinetics of sulfatide in kidney transplant recipients for the first time. We also analyzed the correlated factors influencing the serum sulfatide level, using multiple regression analysis. 


\section{Methods}

\section{Kidney transplant recipients and study protocol}

We studied 5 ESRD adult patients (4 males, 1 female), who underwent kidney transplant at the Shinshu University Hospital from 2007 to 2009. We collected serum samples before and throughout the observation period of kidney transplantation. Serum samples in the HD period were obtained prior to each HD session. We measured the serum level of sulfatide at each point, as well as many other laboratory data, and analyzed these data statistically. For the calculation of the estimated glomerular filtration rate (eGFR), we used the novel 3-variable Japanese equation [11]. Serum lipids including total cholesterol (TC), low density lipoprotein (LDL), high density lipoprotein (HDL), and triglyceride (TG) were measured using commercial enzyme assay kits purchased from Kyowa Medex (Tokyo, Japan), Sekisui Medical (Tokyo, Japan), Daiichi Chemical (Tokyo, Japan), and Mizuho Medy (Saga, Japan), respectively. The Helsinki Declaration, as revised in 2004, was applied to this study. The protocol of this study was approved by the institutional review board of the ethics committee at Shinshu University Hospital. Written informed consent was obtained from each patient before participating in this study.

\section{Quantization of sulfatide in serum}

Sulfatide was extracted from serum according to the hexane/isopropanol method and was converted to lysosulfatide (LS; sulfatide without fatty acids) by saponification with sodium hydroxide [12-17]. After purification, the LS samples were desalted by Mono-tip C18 cartridges (GL Sciences, Tokyo, Japan) followed by the addition of N-acetyl LS possessing sphinganine (LS-d18:0 NAc) as the internal standard. They were then analyzed by matrix-assisted laser desorption ionization-time of flight mass spectrometry (MALDI-TOF MS) using a Voyager Elite XL Biospectrometry Workstation 
(PerSeptive Biosystems, Framingham, MA, USA) [13, 14]. A nitrogen laser (337 nm) was used for ionization, and we employed negative ion mode detection. A two-point external calibration was performed with LS-d18:0 NAc ([M-H]-, 584.31) and LS-(4E)-sphingenine (LS-d18:1) ([M-H]-, 540.28). LS-d 18:1 and LS-d 18:0 NAc were chemically prepared from sulfatide using a deacylation procedure as described in our previous study [14]. Seven molecular species of LS were detected, based on the differences in sphingoid base structure: LS-sphingadienine (LS-d18:2), LS-d18:1, LS-d18:0, LS-phytosphingosine (LS-t18:0), LS-(4E)-icosasphingenine (LS-d20:1), LS-icosasphinganine (LS-d20:0), and LS-4D-hydroxyicosasphinganine (LS-t20:0) [13]. The level of sulfatide was calculated as the sum of the levels of these seven LS species.

\section{Analysis of serum oxidative stress marker}

The serum content of the oxidative stress marker malondialdehyde (MDA) was measured using a lipid peroxidation colorimetric assay kit purchased from Oxis International (Beverly Hills, CA, USA).

\section{Statistical analysis}

Data are reported as the mean $\pm \mathrm{SD}$. Factors that might contribute to the alteration of the serum sulfatide level were analyzed by stepwise multiple regression analysis. Data analysis was performed with the computer program SPSS ver. 18.0J (SPSS, Inc., Chicago, IL, USA). The level of statistical significance was set at $\mathrm{P}<0.05$. Significant differences between the parameters measured before (HD period) and after kidney transplantation (final observation point) were analyzed by a paired $t$ test. 


\section{Results}

\section{Baseline characteristics of kidney transplant recipients}

The ages of kidney transplant recipients on the day of kidney transplant operation ranged from 23 to 57 years old (Table 1 , mean $\pm \mathrm{SD}, 43.4 \pm 13.9$ years). The primary kidney diseases of these recipients were diverse; however, diabetic nephropathy was not included. Most patients had undergone HD prior to kidney transplant. One patient continued peritoneal dialysis therapy (PD) for 102 months, and he then switched from PD to HD 31 months before the kidney transplant operation. Hypertension was present at a relatively high incidence level (60\%), but the incidence of other complications, including impaired glucose tolerance, hyperlipidemia, and hyperuricemia, was low. These complications were mild and well controlled by supplementation with oral medicine. None of the recipients had CVD, including low cardiac function, ischemic heart disease, aortic disease, cerebral infarction and hemorrhage, and peripheral vascular disease. No major organ injury except for kidney failure was detected, indicating the general condition of these recipients was relatively good. Three recipients received the transplant kidney from living donors, and the other 2 recipients from cadaveric donors. Four major immunosuppression agents, including basiliximab, prednisolone, calcineurin inhibitor (cyclosporine A or tacrolimus), and mycophenolate mofetil, were routinely used for kidney transplant protocol. One to three human leukocyte antigens of these recipients differed from those of donors. The mean duration of the observation period after the transplant operation was $566 \pm 364$ days.

There was no operative trouble during any of the kidney transplant operations, and all kidney grafts functioned sufficiently within 1 month.

\section{Clinical course of kidney transplant and alteration of serum level of sulfatide}

Acute rejection did not occur for any recipient during the observation period. The urine volume 
was increased and the serum level of creatinine (Cre) was dramatically decreased after the kidney transplant operation in all recipients within 1 month, after which the serum Cre level became stable (Fig. 1). This data indicated that there are two phases in the recovery process of kidney function, namely the acute phase and the stable phase, suggesting the necessity of separation of the two phases for the analysis of each clinical parameter. To distinguish the two phases of each recipient, we determined the threshold level of creatinine (Table 2). The serum creatinine level any time after the 100th day after the kidney transplant operation of each recipient (mean $+1.96 \mathrm{SD}$ ) was used as a threshold level separating the two phases of kidney function recovery. We defined the period before the serum creatinine level reached to the threshold level, as the acute phase. We defined also the period after the serum creatinine level reached to the threshold level, as the stable phase. Before the kidney transplant operation, the serum levels of sulfatide of each recipient were much lower than those of normal subjects, as published in our earlier study $(2.36 \pm 0.79 \mathrm{vs.} 8.21 \pm 1.50 \mathrm{nmol} / \mathrm{mL})[9]$. After the kidney transplant, the serum levels of sulfatide were dramatically increased in all recipients in a time-dependent manner; however, the recovery speed was slower than that of the improvement of kidney function and the serum sulfatide reached a nearly normal level after 1 year (Fig. 1). This recovery curve seemed to be logarithmic, and the data of serum sulfatide exhibited close linearity with the logarithm of duration (log duration) (Fig. 1). Therefore, we used the log duration as the time parameter for further analyses. The level of sulfatide was calculated as the sum of the levels of seven LS species. We then checked the alteration of serum sulfatide composition that occurred in association with kidney transplantation (Table 3). The percentages of molecular species of LS before (a point in the HD period) and after kidney transplantation (final observation point) were compared, and no significant difference was found, suggesting that kidney transplantation would cause marked 
quantitative changes of the serum sulfatide, but not qualitative changes. Since sulfatide is a major component of glycosphingolipids in lipoproteins, we also checked the alteration of serum lipids including TC, LDL, HDL, and TG. There was a tendency for these lipids to increase after kidney transplantation; however there was no significant difference between the levels measured before and after kidney transplantation, suggesting that the marked increase of serum sulfatide level would be independent of lipoprotein metabolism. In these clinical courses, no marked difference was detected between the grafts from living donors and those from cadaveric donors. These data are the first demonstration that the reduction level of serum sulfatide in ESRD patients was significantly recovered via the kidney transplant process.

\section{Clinical parameters correlating to the serum level of sulfatide}

We next investigated the clinical parameters correlating to the serum levels of sulfatide in the acute and stable phases of kidney function recovery. Our earlier study using a murine AKI model suggested that systemic oxidative stress might influence the serum sulfatide level [10], so we checked a representative oxidative stress marker, the serum level of MDA, as well as the time parameter and the conventional serum parameters. Spearman's rank correlation coefficient between the serum sulfatide level and each parameter was calculated, and possibly significant correlated factors were selected (Table 4). Candidates for significant correlated factors in the acute phase were the log duration, MDA, eGFR, serum level of Cre, chloride, C-reactive protein, and $\gamma$-glutamyl transpeptidase. The scatter plot between the serum sulfatide level and each parameter in acute phase indicated the close linearity of the serum sulfatide level with log duration and MDA (Fig. 2). The other parameters were distributed relatively widely. Candidates of significant correlated factors in the stable phase were the log duration, MDA, serum level of sodium, uric acid, alanine transaminase, hematocrit, and platelet count (PLT) 
(Table 4). The scatter plot between the serum sulfatide level and each parameter in the stable phase indicated the close linearity of the serum sulfatide level with these correlated factors (Fig. 3).

Finally, we used stepwise multiple regression analysis to analyze the factors that might contribute to the alteration of the serum sulfatide level in each phase. In the acute phase, the only significant correlated factor was log duration with a high standardized regression coefficient (Table 5). In the stable phase, there are three significant correlated factors, log duration, MDA, and PLT (Table 5). The intensity of correlation was indicated via the absolute values of the standardized regression coefficient and $\mathrm{P}$ value, suggesting the strongest correlated factor was log duration, followed by reduction of MDA, followed by increase of PLT. 


\section{Discussion}

There have been few studies investigating the alteration of the human serum level of sulfatide accompanying organ dysfunction, or of the related factors influencing the serum sulfatide level. Therefore, we hope that the results of the present study investigating the alteration of the serum sulfatide level in kidney transplant recipient will be valuable when considering the kinetics of sulfatide in human serum. Our earlier studies investigating HD patients demonstrated the diminished level of serum sulfatide under a kidney dysfunctional state [9]. However, it was unknown whether the reduction of human serum sulfatide level was directly caused by kidney dysfunction and whether this alteration was recovered by compensation of kidney function. The present study demonstrated for the first time that recovery of the human serum sulfatide level occurred via the kidney transplant procedure, suggesting that the human serum sulfatide level is closely affected by kidney function.

Since the biosynthesis of sulfatide species is regulated by tissue-dependent desaturases and hydroxylases, its composition differs in important organs, such as the liver and kidney [4]. Therefore, a similarity in the percentage of sulfatide species between serum and an organ would provide important information concerning the source of serum sulfatide. The current study demonstrated that the serum sulfatide composition was scarcely changed via kidney transplantation, suggesting that the contribution of tissue-dependent desaturases and hydroxylases were not influenced by kidney transplantation. Our earlier experimental study using protein-overload nephropathy, a type of AKI murine model, indicated the considerable reduction of the serum sulfatide level in response to the AKI process [10]. Interestingly, this animal study showed the similarity of sphingoid composition of serum and hepatic sulfatide, and the synchronized reduction of the serum level and the hepatic content of sulfatide. On the other hand, the sphingoid composition of renal sulfatide in the AKI mouse differed from those of serum 
sulfatide, and the content of renal sulfatide was not decreased. These experimental results revealed that alteration of the serum sulfatide level via kidney dysfunction is dependent on the hepatic secretory ability of sulfatide. Another experimental study using rabbits also showed that the sulfatide of serum lipoproteins was mostly derived from the liver [4]. These results suggest that the source of increased sulfatide in kidney transplant recipients is the liver. Today, we have no information concerning the sulfatide composition in the livers of kidney transplant recipients, so we need to obtain this information to confirm our hypothesis in the future.

The current study showed the slow recovery of serum sulfatide level following the improvement of kidney function in kidney transplant recipients. Most of the serum conditions experienced by kidney transplant recipients, such as the accumulation of uremic toxin and the instability of various important balances, including body fluid volume, electrolytes, and acid base balance, became generally stabilized within 1 month after the transplant operation. The reduction of serum sulfatide was attenuated in this acute phase, though it did not reach the normal level, and then it gradually recovered over a long time period. This phenomenon suggested that the improvement of kidney function affected the serum sulfatide level through an indirect mechanism. Interestingly, the current study demonstrated the significant correlation between the serum oxidative stress marker, MDA, and the serum sulfatide level, especially in the stable phase. In human kidney transplant recipients, improvement of kidney function would reduce systemic oxidative stress at first, and then this good internal environmental condition might increase the hepatic ability to secrete sulfatide. This speculation is supported by our two murine experimental studies. First, the results of the experimental study of AKI murine model demonstrated that kidney dysfunction induced systemic oxidative stress followed by the down-regulation of hepatic expression of cerebroside sulfotransferase (CST), an important key enzyme in sulfatide synthesis, 
resulting in the reduction of serum sulfatide level [10]. Second, our unpublished experimental study investigating ethanol effects demonstrated the relationship between oxidative stress and hepatic sulfatide synthesis independent of kidney dysfunction. In this ethanol study, we examined serum and liver sulfatide levels in mice that ingested ethanol ( $500 \mathrm{mg} / \mathrm{kg}$ body weight) for 21 days ( $\mathrm{n}=6 \mathrm{vs.} .8$ for control and ethanol-treated mice, respectively). The ethanol treatment resulted in the increase of a representative oxidative stress marker, the hepatic tissue amount of MDA+4-hydroxynonenal (MDA/4-HNE, $0.062 \pm 0.013$ vs. $0.143 \pm 0.032 \mathrm{nmol} / \mathrm{hg}$ liver tissue, for control and ethanol-treated mice, respectively), and the decrease of the sulfatide level in the serum and liver (serum sulfatide level, $1.50 \pm 0.14$ vs. $1.06 \pm 0.09 \mathrm{nmol} / \mathrm{mL}$; hepatic sulfatide amount, $34.34 \pm 6.78$ vs. $15.67 \pm 2.89 \mathrm{pmol} / \mathrm{mg}$ for control and ethanol-treated mice, respectively), suggesting a close relation between oxidative stress and hepatic sulfatide synthesis. In the future, the serum sulfatide levels of human patients under severe oxidative stress due to various systemic diseases should be examined to confirm our speculation.

Since serum sulfatide exists in lipoproteins, there is a possibility that the attenuation of oxidative stress resulting from kidney transplantation improves the hepatic ability to secrete sulfatide through the alteration of the lipoprotein metabolism. Since it was reported that sulfatide is contained equally in various lipoproteins, including chylomicrons, VLDL, LDL, and HDL [4], we evaluated the alteration of representative serum lipid levels in kidney transplant recipients. The current study showed an insignificant alteration of the serum lipid profile due to kidney transplantation, suggesting that lipoprotein metabolism makes little contribution to the marked increase of the serum sulfatide level in kidney transplant recipients.

In the current study, another important correlation between PLT and the serum sulfatide level in human kidney transplant recipients was revealed for the first time. Many past studies demonstrated the 
role of sulfatide in influencing platelet aggregation [6]. Sulfatide is known to bind with adhesive proteins such as laminin, thrombospondin, von Willebrand factor (vWF), P-selectin, and fibrinogen $[8$, 18-21]. Laminin is a major component of the basement membrane, and it mediates platelet adhesion via laminin receptors on platelets, glycoproteins (GP) Ic/IIa [22]. Thrombospondin, which is a component of the extracellular matrix and the most abundant constituent in platelet $\alpha$-granules, promoted platelet adhesion [19]. vWF is a glycoprotein that is the most important bridging component of subendothelium and platelet receptor sites on GP Ib and IIb/IIIa [20]. P-selectin, which is also present in the $\alpha$-granules, stabilizes GP IIb/IIIa-fibrinogen interaction and forms stable platelet aggregates [23]. Sulfatide is expressed on the platelet surface, and the expressing sulfatide interacts with these multi molecules, and then contributes to platelet aggregates $[6,21]$. It was reported that exogenous sulfatide inhibited the platelet aggregations in an in vitro assay, and that intravenous administration of exogenous sulfatide prolonged bleeding time $[8,21,24]$. Moreover, sulfatide exerts anti-coagulant effects through their direct binding to fibrinogen [8]. These findings indicate the anti-platelet and anti-coagulant effect of sulfatide in serum, blocking the interaction between membranous sulfatide expressed on platelets and various adhesion molecules. The current study demonstrated the positive correlation between PLT and the serum sulfatide level. This result suggests that the increase of serum sulfatide observed in kidney transplant recipients might affect to the platelet count and the activity of platelet, resulting in anti-platelet effects. The many epidemiologic studies investigating ESRD patients demonstrate that CVD mortality rates of kidney transplant recipients are significantly lower than that of the age-matched HD population [25]. This favorable effect of kidney transplant might be partially derived from the anti-platelet effect via recovery of the serum sulfatide level.

In conclusion, the current study results demonstrated the gradual but reliable recovery of the 
serum sulfatide level in kidney transplant recipients. This recovery might be derived from the attenuation of systemic oxidative stress caused by chronic kidney dysfunction. The normalized serum sulfatide level might exert anti-platelet effects, and contribute to a reduced incidence of CVD. However, the current study was an observational study, in which we investigated a small number of kidney transplant recipients in a single institution, and therefore a larger-scale multi-center clinical study will be needed to confirm our results. Furthermore, more evidence provided by clinical and biological studies will be needed to confirm our speculation concerning the function of serum sulfatide in humans.

\section{Acknowledgment}

This work was supported in part by GL Sciences (Tokyo, Japan) and Shinshu Public Utility Foundation for the Promotion of Medical Sciences (Matsumoto, Japan). 


\section{References}

1. Ishizuka, I.: Chemistry and functional distribution of sulfoglycolipids. Prog. Lipid Res. 36, 245-319 (1997)

2. Honke, K., Zhang, Y., Cheng, X., Kotani, N., Taniguchi, N.: Biological roles of sulfoglycolipids and pathophysiology of their deficiency. Glycoconj. J. 21, 59-62 (2004)

3. Nagai, K., Tadano-Aritomi, K., Niimura, Y., Ishizuka, I.: Higher expression of renal sulfoglycolipids in marine mammals. Glycoconj. J. 25, 723-726 (2008)

4. Hara, A., Taketomi, T.: Occurrence of sulfatide as a major glycosphingolipid in WHHL rabbit serum lipoproteins. J. Biochem. 102, 83-92 (1987)

5. Zhu, X.H., Hara, A., Taketomi,T.: The existence of galactosylceramide $\mathrm{I}^{3}$-sulfate in serums of various mammals and its anticoagulant activity. J. Biochem. 110, 241-245 (1991)

6. Kyogashima, M.: The role of sulfatide in thrombogenesis and haemostasis. Arch. Biochem. Biophys. 426, 157-162 (2004)

7. Hara, A., Taketomi, T.: Characterization and changes of glycosphingolipids in the aorta of the Watanabe hereditable hyperlipidemic rabbit. J. Biochem. 109, 904-908 (1991)

8. Hara, A., Uemura, K., Taketomi, T.: Sulfatide prolongs blood-coagulation time and bleeding time by forming a complex with fibrinogen. Glycoconj. J. 13, 187-194 (1996)

9. Hu, R., Li, G., Kamijo, Y., Aoyama, T., Nakajima, T., Inoue, T., Node, K., Kannagi, R., Kyogashima, M., Hara, A.: Serum sulfatides as a novel biomarker for cardiovascular disease in patients with end-stage renal failure. Glycoconj. J. 24, 565-571 (2007)

10. Zhang, X., Nakajima, T., Kamijo, Y., Li, G., Hu, R., Kannagi, R., Kyogashima, M., Aoyama, T., Hara, A.: Acute kidney injury induced by protein-overload nephropathy down-regulates 
gene expression of hepatic cerebroside sulfotransferase in mice, resulting in reduction of liver and serum sulfatides. Biochem. Biophys. Res. Commun. 390, 1382-1388 (2009)

11. Matsuo, S., Imai, E., Horio, M., Yasuda, Y., Tomita, K., Nitta, K., Yamagata, K., Tomino, Y., Yokoyama, H., Hishida, A., Collaborators developing the Japanese equation for estimated GFR: revised equations for estimated GFR from serum creatinine in Japan. Am. J. Kidney Dis. 53, 982-992 (2009)

12. Hara, A., Radin, N.S.: Lipid extraction of tissues with a low-toxicity solvent. Anal. Biochem. 90, 420-426 (1978)

13. Li, G., Hu, R., Kamijo, Y., Nakajima, T., Aoyama, T., Inoue, T., Node, K., Kannagi, R., Kyogashima, M., Hara, A.: Establishment of a quantitative, qualitative, and high-throughput analysis of sulfatides from small amounts of sera by matrix-assisted laser desorption ionization-time of flight mass spectrometry. Anal. Biochem. 362, 1-7 (2007)

14. Sugiyama, E., Hara, A., Uemura, K.: A quantitative analysis of serum sulfatide by matrix-assisted laser desorption ionization time-of-flight mass spectrometry with delayed ion extraction. Anal. Biochem. 274, 90-97 (1999)

15. Aoyama, T., Peters, J.M., Iritani, N., Nakajima, T., Furihata, K., Hashimoto, T., Gonzalez, F.J.: Altered constitutive expression of fatty acid-metabolizing enzymes in mice lacking the peroxisome proliferator-activated receptor alpha (PPARalpha). J. Biol. Chem. 273, 5678-5684 (1998)

16. Aoyama, T., Uchida, Y., Kelley, R.I., Marble, M., Hofman, K., Tonsgard, J.H., Rhead, W.J., Hashimoto, T.: A novel disease with deficiency of mitochondrial very-long-chain acyl-CoA dehydrogenase. Biochem. Biophys. Res. Commun. 191, 1369-1372 (1993) 
17. Aoyama, T., Yamano, S., Waxman, D.J., Lapenson, D.P., Meyer, U.A., Fischer, V., Tyndale, R., Inaba, T., Kalow, W., Gelboin, H.V., Gonzalez, F.J.: Cytochrome P-450 hPCN3, a novel cytochrome P-450 IIIA gene product that is differentially expressed in adult human liver. cDNA and deduced amino acid sequence and distinct specificities of cDNA-expressed hPCN1 and hPCN3 for the metabolism of steroid hormones and cyclosporine. J. Biol. Chem. 264, 10388-10395 (1989)

18. Roberts, D.D., Rao, C.N., Magnani, J.L., Spitalnik, S.L., Liotta, L.A., Ginsburg, V.: Laminin binds specifically to sulfated glycolipids. Proc. Natl. Acad. Sci. U.S.A. 82, 1306-1310 (1985)

19. Roberts, D.D., Haverstick, D.M., Dixit, V.M., Frazier, W.A., Santoro, S.A., Ginsburg, V.: The platelet glycoprotein thrombospondin binds specifically to sulfated glycolipids. J. Biol. Chem. 260, 9405-9411 (1985)

20. Roberts, D.D., Williams, S.B., Gralnick, H.R., Ginsburg, V.: Von Willebrand factor binds specifically to sulfated glycolipids. J. Biol. Chem. 261, 3306-3309 (1986)

21. Merten, M., Thiagarajan, P.: Role for sulfatides in platelet aggregation. Circulation. 104, $2955-2960(2001)$

22. Jones, J.C., Dehart, G.W., Gonzales, M., Goldfinger, L.E.: Laminins: an overview. Microsc. Res. Tech. 51, 211-213 (2000)

23. Hawiger, J.: Mechanisms involved in platelet vessel wall interaction. Thromb. Haemost. 74, 369-372 (1995)

24. Borthakur, G., Cruz, M.A., Dong, J.F., McIntire, L., Li, F., López, J.A., Thiagarajan, P.: Sulfatides inhibit platelet adhesion to von Willebrand factor in flowing blood. J. Thromb. Haemost. 1, 1288-1295 (2003) 
25. Sarnak, M.J., Levey, A.S., Schoolwerth, A.C., Coresh, J., Culleton, B., Hamm, L.L., McCullough, P.A., Kasiske, B.L., Kelepouris, E., Klag, M.J., Parfrey, P., Pfeffer, M., Raij, L., Spinosa, D.J., Wilson, P.W., American Heart Association Councils on Kidney in Cardiovascular Disease, High Blood Pressure Research, Clinical Cardiology, and Epidemiology and Prevention.: Kidney disease as a risk factor for development of cardiovascular disease: a statement from the American Heart Association Councils on Kidney in Cardiovascular Disease, High Blood Pressure Research, Clinical Cardiology, and Epidemiology and Prevention. Circulation 108, 2154-2169 (2003) 


\section{Figure legends}

\section{Fig. 1}

Time course of serum concentration of creatinine and sulfatide after kidney transplantation. The data of serum sulfatide exhibited close linearity with the logarithm of duration (log duration). The red triangles and blue circles indicate the data obtained from recipients whose kidney grafts were supplied by living donors and cadaveric donors, respectively. The reduction level of serum sulfatide in ESRD patients was significantly recovered via kidney transplantation. The recovery speed of serum sulfatide level was slower than that of the improvement of kidney function and the serum sulfatide reached a nearly normal level after 1 year.

\section{Fig. 2}

The scatter plot for the acute phase of kidney transplantation indicates the relationship between the serum sulfatide level and correlated factors selected by analysis of Spearman's rank correlation coefficient. The red triangles and blue circles indicate the data obtained from recipients whose kidney grafts were supplied by living donors and cadaveric donors, respectively. There is close linearity of the serum sulfatide level with log duration and MDA. The other parameters were distributed relatively widely.

\section{Fig. 3}

The scatter plot for the stable phase indicates the relationship between the serum sulfatide level and correlated factors. The red triangles and blue circles indicate the data obtained from recipients whose kidney grafts were supplied by living donors and cadaveric donors, respectively. There is close linearity of the serum sulfatide level with these correlated factors. 
Table 1. Baseline characteristics of kidney transplant recipients.

\begin{tabular}{|c|c|c|c|c|c|}
\hline Patient Number & 1 & 2 & 3 & 4 & 5 \\
\hline Age (years) & 36 & 57 & 53 & 48 & 23 \\
\hline Sex & $\mathrm{M}$ & $\mathrm{F}$ & $\mathrm{M}$ & $\mathrm{M}$ & $\mathrm{M}$ \\
\hline Primary disease & PCK & FGS & $\mathrm{CP}$ & CGN & $\operatorname{IgANP}$ \\
\hline $\begin{array}{c}\text { Medication history } \\
\text { (HD or PD) }\end{array}$ & HD & $\mathrm{HD}$ & HD & $\mathrm{PD} / \mathrm{HD}$ & HD \\
\hline $\begin{array}{l}\text { HD or PD duration } \\
\text { (months) }\end{array}$ & 7.5 & 296 & 108 & $\begin{array}{l}\text { PD } 102 \\
\text { HD } 31\end{array}$ & 16 \\
\hline \multicolumn{6}{|l|}{ Complication } \\
\hline $\mathrm{HT}$ & + & + & + & - & - \\
\hline IGT & - & - & + & - & - \\
\hline Hyperlipidemia & - & - & - & - & - \\
\hline Hyperuricemia & + & - & - & - & - \\
\hline CVD & - & - & - & - & - \\
\hline $\begin{array}{l}\text { Donor information } \\
\text { (living or cadaveric) }\end{array}$ & LD & $\mathrm{CD}$ & LD & $\mathrm{CD}$ & LD \\
\hline \multicolumn{6}{|l|}{ Immunosuppression } \\
\hline Basiliximab & + & + & + & + & + \\
\hline PSL & + & + & + & + & + \\
\hline Cyclosporine A & + & - & - & - & - \\
\hline Tacrolimus & - & + & + & + & + \\
\hline MMF & + & + & + & + & + \\
\hline HLA mismatch & 3 & 2 & 3 & 3 & 1 \\
\hline $\begin{array}{l}\text { Observation period } \\
\text { after transplant (days) }\end{array}$ & 1098 & 782 & 217 & 379 & 356 \\
\hline
\end{tabular}

CGN, chronic glomerulonephritis; CP, chronic pyelonephritis; CVD, cardiovascular disease; FGS, focal glomerulosclerosis; HD hemodialysis; HLA, human leukocyte antigen; HT, hypertension; IgA NP, IgA nephropathy; IGT, impaired glucose tolerance; MMF, mycophenolate mofetil; PCK, polycystic kidney disease; PD peritoneal dialysis; PSL, prednisolone 
Table 2. Threshold levels of creatinine of each patient, separating the acute phase from the stable phase.

The serum creatinine level any time after the 100th day after the kidney transplant

\begin{tabular}{cccc}
\hline Patient No. & Mean \pm SD $(\mathrm{mg} / \mathrm{dl})$ & 1.96 SD & Threshold level \\
\hline 1 & $1.020 \pm 0.127$ & 0.249 & 1.269 \\
2 & $1.258 \pm 0.190$ & 0.372 & 1.630 \\
3 & $1.313 \pm 0.046$ & 0.090 & 1.403 \\
4 & $2.075 \pm 0.191$ & 0.375 & 2.450 \\
5 & $1.543 \pm 0.079$ & 0.155 & 1.698 \\
\hline
\end{tabular}

operation (mean $+1.96 \mathrm{SD}$ of each recipient) was used as a threshold level for each patient. 
Table 3. The effects of kidney transplantation against serum sulfatide composition and lipids level.

\begin{tabular}{cccc}
\hline & $\begin{array}{c}\text { Before KT } \\
(\text { Mean } \pm \text { SD) }\end{array}$ & $\begin{array}{c}\text { After KT } \\
(\text { Mean } \pm \text { SD) }\end{array}$ & P-value \\
\hline Lysosulfatide species & & & \\
LS-d 18:2 (\%) & $13.8 \pm 2.8$ & $15.4 \pm 1.7$ & 0.289 \\
LS-d 18:1 (\%) & $57.3 \pm 6.6$ & $54.7 \pm 8.4$ & 0.577 \\
LS-d 18:0 (\%) & $5.0 \pm 2.8$ & $6.7 \pm 1.4$ & 0.095 \\
LS-t 18:0 (\%) & $11.6 \pm 2.9$ & $8.4 \pm 4.3$ & 0.263 \\
LS-d 20:1 (\%) & $3.6 \pm 5.1$ & $5.1 \pm 5.1$ & 0.324 \\
LS-d 20:0 (\%) & $5.3 \pm 2.7$ & $7.0 \pm 4.0$ & 0.492 \\
LS-t 20:0 (\%) & $3.5 \pm 1.8$ & $2.8 \pm 2.6$ & 0.655 \\
\hline Serum lipids & & & \\
TC (mg/dL) & $145 \pm 70$ & $166 \pm 56$ & 0.079 \\
LDL (mg/dL) & $85 \pm 39$ & $95 \pm 32$ & 0.111 \\
HDL (mg/dL) & $45 \pm 24$ & $50 \pm 26$ & 0.106 \\
TG (mg/dL) & $95 \pm 33$ & $110 \pm 48$ & 0.325 \\
\hline
\end{tabular}

HDL, high density lipoprotein; KT, kidney transplantation; LDL, low density lipoprotein; TC, total cholesterol; TG, Triglyceride. Mean level of serum sulfatide composition in 5 kidney transplant recipients was indicated as a percentage of total lysosulfatides, which were measured by MALDI-TOF MS. Significant difference between two points, before and after kidney transplantation, were analyzed by paired $t$ test. 
Table 4. Spearman correlation between serum sulfatide level and each paramater.

\begin{tabular}{|c|c|c|c|c|}
\hline & $\begin{array}{c}\text { Acute } \\
(\text { Mean } \pm \mathrm{SD})\end{array}$ & $\begin{array}{l}\text { Spearman } \\
\text { correlation }\end{array}$ & $\begin{array}{c}\text { Stable } \\
(\text { Mean } \pm \mathrm{SD})\end{array}$ & $\begin{array}{c}\text { Spearman } \\
\text { correlation }\end{array}$ \\
\hline Sulfatide $(\mathrm{nmol} / \mathrm{mL})$ & $2.73 \pm 0.75$ & 1 & $4.91 \pm 1.71$ & 1 \\
\hline Log duration & $0.97 \pm 0.56$ & $0.854 * * *$ & $1.96 \pm 0.71$ & $0.928 * *$ \\
\hline MDA $(\mu \mathrm{mol} / \mathrm{L})$ & $1.70 \pm 0.55$ & $-0.618 * *$ & $1.51 \pm 0.65$ & $-0.899 * *$ \\
\hline $\operatorname{eGFR}\left(\mathrm{ml} / \mathrm{min} / 1.73 \mathrm{~m}^{2}\right)$ & $18.03 \pm 14.66$ & $0.471 *$ & $44.15 \pm 12.50$ & -0.334 \\
\hline Total protein (g/dL) & $6.14 \pm 0.83$ & 0.079 & $7.03 \pm 0.71$ & 0.145 \\
\hline Albumin (g/dL) & $3.56 \pm 0.54$ & -0.029 & $4.24 \pm 0.38$ & 0.219 \\
\hline BUN (mg/dL) & $37.25 \pm 15.68$ & -0.04 & $20.64 \pm 9.22$ & 0.237 \\
\hline Creatinine (mg/dL) & $5.92 \pm 4.49$ & $-0.536^{* * *}$ & $1.54 \pm 0.40$ & 0.147 \\
\hline Sodium (mEq/L) & $137.96 \pm 3.24$ & 0.081 & $138.48 \pm 3.08$ & $0.437^{*}$ \\
\hline Potassium (mEq/L) & $4.14 \pm 0.50$ & -0.322 & $4.30 \pm 0.44$ & -0.217 \\
\hline Chloride (mEq/L) & $105.07 \pm 5.86$ & $0.419 *$ & $106.03 \pm 2.78$ & -0.147 \\
\hline Calcium (mg/dL) & $8.46 \pm 0.60$ & 0.083 & $9.49 \pm 0.45$ & 0.324 \\
\hline CRP $\quad(\mathrm{mg} / \mathrm{dL})$ & $0.77 \pm 1.10$ & $-0.399 *$ & $0.17 \pm 0.41$ & 0.069 \\
\hline Uric acid (mg/dL) & $5.01 \pm 1.46$ & 0.252 & $6.52 \pm 1.46$ & $0.529 * *$ \\
\hline $\mathbf{A S T}(\mathrm{IU} / \mathrm{L})$ & $21.24 \pm 9.90$ & -0.094 & $21.85 \pm 9.04$ & -0.402 \\
\hline $\mathbf{A L T}(\mathrm{IU} / \mathrm{L})$ & $26.05 \pm 14.85$ & 0.327 & $23.3 \pm 11.84$ & $-0.534^{*}$ \\
\hline$\gamma \mathbf{G T P}(\mathrm{IU} / \mathrm{L})$ & $64.95 \pm 79.88$ & $0.685^{* *}$ & $35.7 \pm 15.72$ & 0.291 \\
\hline T-bil (mg/dL) & $0.80 \pm 0.50$ & 0.31 & $0.84 \pm 0.36$ & 0.014 \\
\hline $\mathbf{A L P}(\mathrm{IU} / \mathrm{L})$ & $323.5 \pm 177.0$ & 0.028 & $311.1 \pm 162.5$ & -0.25 \\
\hline LDH (IU/L) & $239.4 \pm 127.1$ & 0.243 & $176.5 \pm 84.1$ & -0.269 \\
\hline WBC (count $/ \mu \mathrm{L})$ & $7527 \pm 3449$ & -0.199 & $5925 \pm 1944$ & -0.317 \\
\hline $\mathbf{R B C}$ (count $\left.\times 10^{4} / \mu \mathrm{L}\right)$ & $340.2 \pm 67.7$ & -0.109 & $441.4 \pm 86.5$ & 0.252 \\
\hline Hemoglobin (g/dL) & $10.31 \pm 1.65$ & -0.003 & $12.60 \pm 2.29$ & 0.321 \\
\hline Hematocrit (\%) & $31.15 \pm 4.88$ & -0.007 & $38.9 \pm 6.18$ & $0.407^{*}$ \\
\hline Platelet (count $\left.\times 10^{4} / \mu \mathrm{L}\right)$ & $18.09 \pm 4.98$ & 0.367 & $18.62 \pm 4.06$ & $0.492 * *$ \\
\hline
\end{tabular}

AST, aspartate amino transferase; ALP, alkaline phosphatase ALT, alanine transaminase; CRP, C-reactive protein; eGFR, estimated glomerular filtration rate; 
LDH, lactate dehydrogenase; $\gamma \mathrm{GTP}, \quad \gamma$-glutamyl transpeptidase; MDA, malondialdehyde; RBC, red blood cell count; T-bil, total bilirubin; WBC, white blood cell count. Spearman's rank correlation coefficient between the serum level of sulfatide and each parameter was calculated. Significance probability is indicated with asterisks $\left({ }^{*} \mathrm{P}<0.05, * * \mathrm{P}<0.01, * * * \mathrm{P}<0.001\right)$. 
Table 5. Multiple linear regression analysis.

Acute phase

\begin{tabular}{|c|c|c|c|c|}
\hline & B & $95 \% \mathrm{CI}$ & $\beta$ & $\mathrm{p}$ \\
\hline Log duration & 1.355 & $0.932 \sim 1.779$ & 0.905 & $<0.001$ \\
\hline \multicolumn{5}{|l|}{ Stable phase } \\
\hline & B & $95 \% \mathrm{CI}$ & $\beta$ & $\mathrm{p}$ \\
\hline Log duration & 1.481 & $0.754 \sim 2.207$ & 0.586 & 0.001 \\
\hline MDA & -0.892 & $-1.697 \sim-0.087$ & -0.318 & 0.033 \\
\hline PLT & 0.095 & $0.013 \sim 0.177$ & 0.238 & 0.026 \\
\hline
\end{tabular}

$\mathrm{B}$, regression coefficient; $\beta$, standardized regression coefficient; $95 \% \mathrm{CI}, 95 \%$ confidence interval of regression coefficient. 

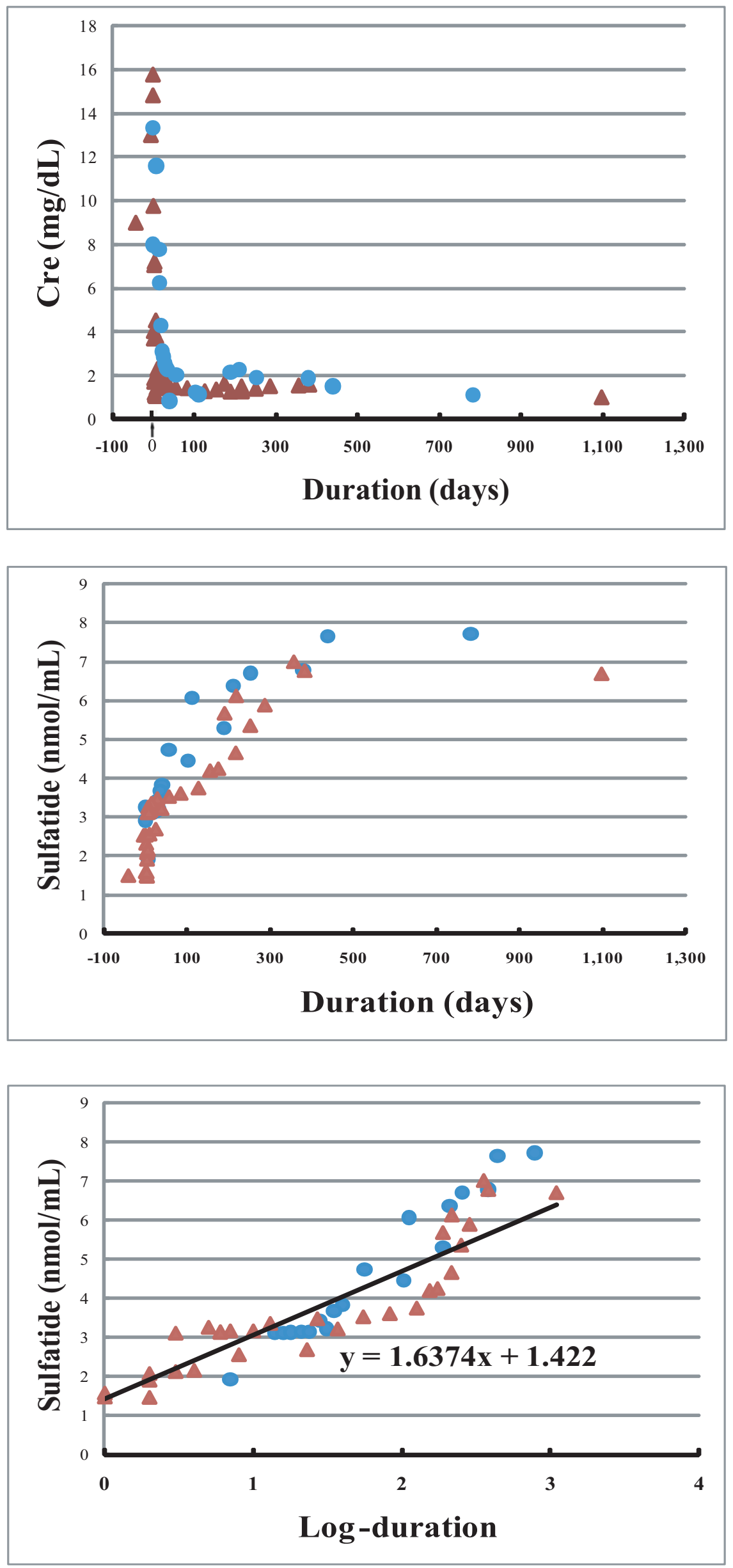

$\Delta$ Living Donor (LD) C Cadaveric Donor (CD)

Fig. 1 
Relationship between serum sulfatide level and each parameter in acute phase
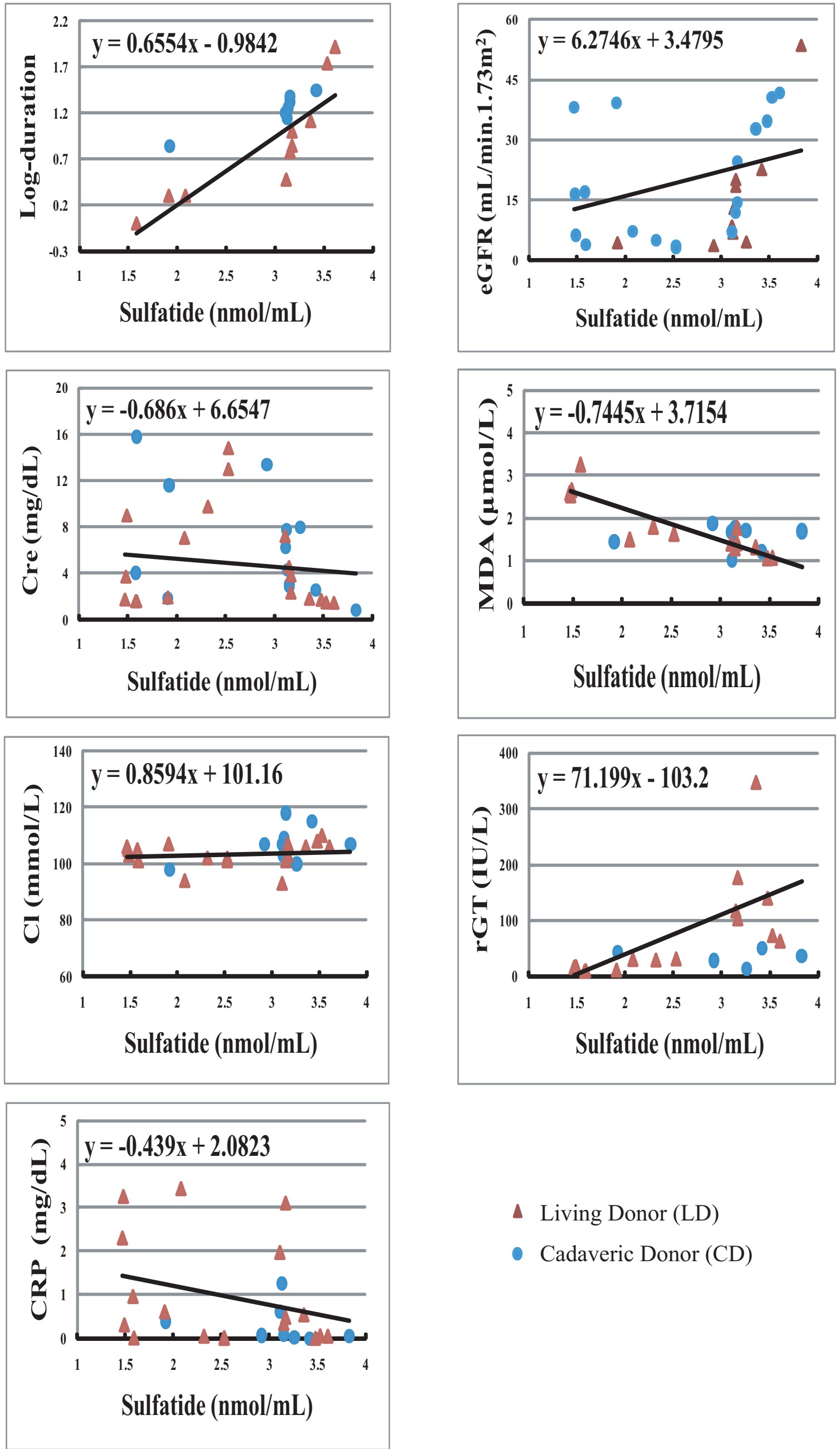

$\triangle$ Living Donor (LD)

- Cadaveric Donor (CD)

Fig. 2 
Relationship between serum sulfatide level and each parameter in stable period
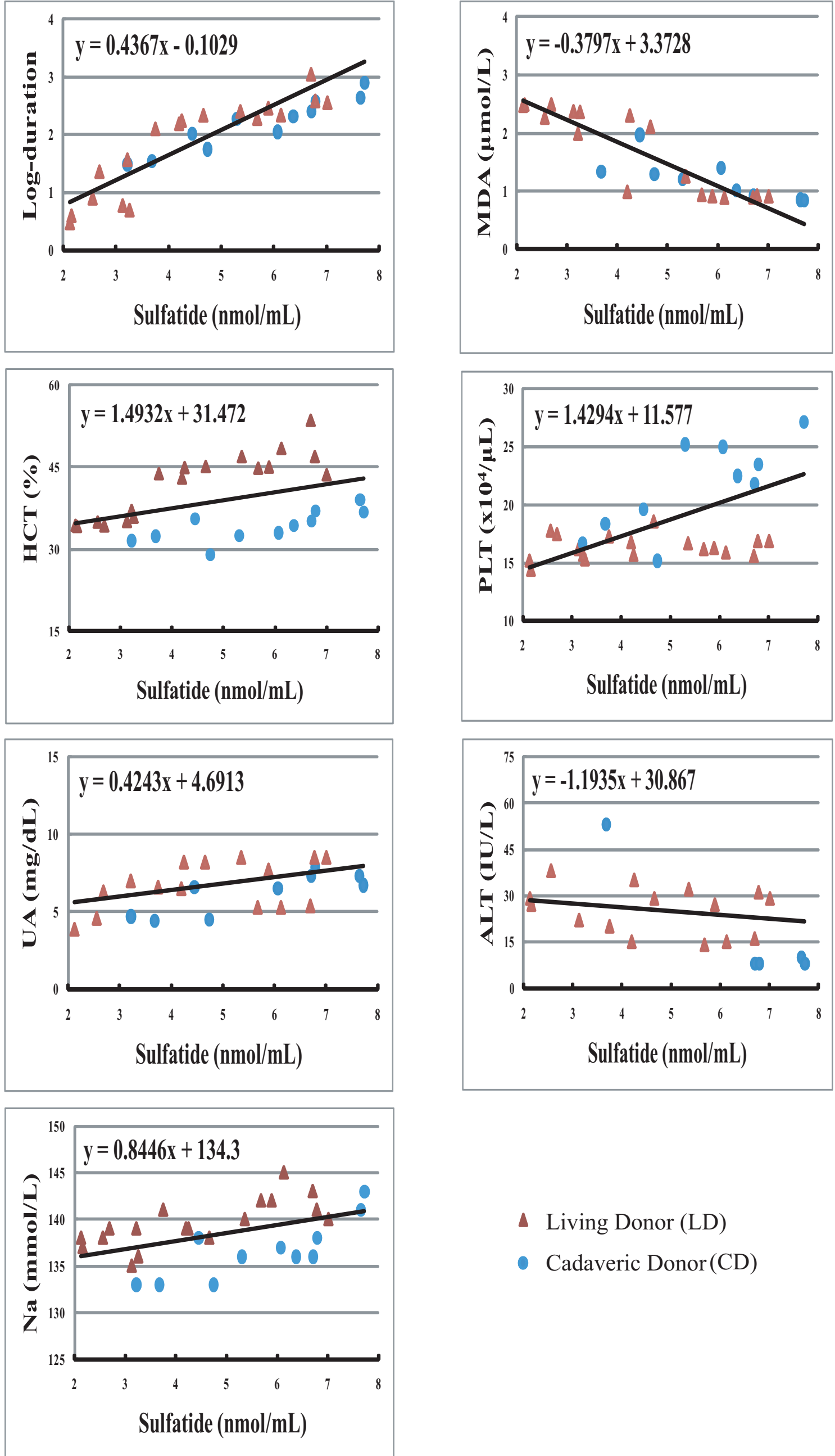
$\triangle$ Living Donor (LD)
- Cadaveric Donor(CD)

Fig. 3 